\title{
Direct endoscopic necrosectomy: a minimally invasive endoscopic technique for the treatment of infected walled-off pancreatic necrosis and infected pseudocysts with solid debris
}

\author{
Tiing Leong Ang ${ }^{1}$, MBBS, FRCP, Andrew Boon Eu Kwek ${ }^{1}$, MBBS, MRCP, Siong San Tan'2, MBBS, FRCS, \\ Salleh Ibrahim ${ }^{2}$, MBBS, FRCS, Kwong Ming Fock ${ }^{1}$, MBBS, FRCP, Eng Kiong Teo $^{1}$, MBBS, FRCP
}

\begin{abstract}
INTRODUCTION Endoscopic transenteric stenting is the standard treatment for pseudocysts, but it may be inadequate for treating infected collections with solid debris. Surgical necrosectomy results in significant morbidity. Direct endoscopic necrosectomy (DEN), a minimally invasive treatment, may be a viable option. This study examined the efficacy and safety of DEN for the treatment of infected walled-off pancreatic necrosis and infected pseudocysts with solid debris. METHODS This study was a retrospective analysis of data collected from a prospective database of patients who underwent DEN in the presence of infected walled-off pancreatic necrosis or infected pseudocysts with solid debris from April 2007 to October 2011. DEN was performed as a staged procedure. Endoscopic ultrasonography-guided transgastric stenting was performed during the first session for initial drainage and to establish endoscopic access to the infected collection. In the second session, the drainage tract was dilated endoscopically to allow transgastric passage of an endoscope for endoscopic necrosectomy. Outcome data included technical success, clinical success and complication rates.

RESULTS Eight patients with infected walled-off pancreatic necrosis or infected pseudocysts with solid debris (mean size $12.5 \mathrm{~cm}$; range $7.8-17.2 \mathrm{~cm}$ ) underwent DEN. Underlying aetiologies included severe acute pancreatitis $(n=6)$ and post-pancreatic surgery $(n=2)$. DEN was technically successful in all patients. Clinical resolution was achieved in seven patients. One patient with recurrent collection opted for surgery instead of repeat endotherapy. No procedural complications were encountered.

CONCLUSION DEN is a safe and effective minimally invasive treatment for infected walled-off pancreatic necrosis and infected pseudocysts.
\end{abstract}

Keywords: debridement, endoscopic drainage, infected pseudocyst, pancreatic necrosis

\section{INTRODUCTION}

Endoscopic ultrasonography (EUS)-guided transenteric stenting is now regarded as the technique of choice for the drainage of symptomatic pancreatic pseudocyst (PC) due to its lower morbidity as compared to surgical and percutaneous drainage, as well as an efficacy similar to that of surgery. ${ }^{(1-3)}$ In the context of infected pancreatic fluid collections with solid debris, inserting transenteric stents alone may be inadequate, as the infected solid debris may require physical removal. In fact, comparative studies have shown that unlike for PC, where the clinical success rate of endoscopic drainage ranged from over $90 \%$ to nearly $100 \%$, transenteric drainage alone for infected walled-off pancreatic necrosis (WOPN) had a much lower success rate of $25 \%-45 \%$. $^{(4,5)}$ Surgical necrosectomy for these patients may result in significant morbidity.

Direct endoscopic necrosectomy (DEN) is a minimally invasive treatment option that has been introduced in recent years for the treatment of infected WOPN. ${ }^{(6,7)}$ Infected WOPN are pancreatic collections that arise as a complication of severe necrotising pancreatitis, where liquefactive necrosis occurs, and in which a pseudo-capsule or wall subsequently develops. With the technique of DEN, a stoma is created endoscopically between the enteric lumen and the walled-off fluid collection to allow the insertion of an endoscope directly into the fluid collection, which allows endoscopic necrosectomy to be performed.

This Singapore study presents our initial experience of DEN via a transgastric approach for the treatment of infected WOPN and infected PC with solid debris, and examines its efficacy and safety.

\section{METHODS}

This study was conducted at the Department of Gastroenterology, Changi General Hospital, Singapore. It was a retrospective analysis of data collected from a prospective database set up when endoscopic drainage of walled-off pancreatic collections was offered as part of the hospital's clinical service in November 2006. All patients were managed by specialists from either the Department of Gastroenterology or Surgery. Gastroenterologists and pancreaticobiliary surgeons collaborated closely, and patients with pancreatic collections, who were deemed to be

${ }^{1}$ Department of Gastroenterology, ${ }^{2}$ Department of Surgery, Changi General Hospital, Singapore

Correspondence: Adj A/Prof Ang Tiing Leong, Chief and Senior Consultant, Department of Gastroenterology, Changi General Hospital, 2 Simei Street 3 , Singapore 529889. tiing_leong_ang@cgh.com.sg 
suitable for endoscopic intervention, were referred for endoscopic treatment to the same gastroenterologist with subspeciality interest in advanced pancreaticobiliary endoscopy. The study adhered to the principles of good clinical practice. Informed consent was obtained from all patients prior to endoscopy.

The definitions of pancreatic fluid collections were based on the revised Atlanta classification by the Acute Pancreatitis Classification Working Group, and are described as follows: ${ }^{(8)}$ (a) acute peripancreatic fluid collections (APFC): acute fluid collections that are not associated with necrosis, and which occur within the first four weeks of acute pancreatitis, have no solid component, are located in or near the pancreas, and always lacks a wall of fibrous or granulation tissue; (b) PC: a collection of pancreatic juice enclosed by a wall of fibrous or granulation tissue as a result of acute pancreatitis, pancreatic trauma or chronic pancreatitis; (c) infected PC: an infected PC contains purulent liquid without an associated solid necrotic component (we recognised that infected PCs may contain solid debris even in the absence of pancreatic necrosis, and hence, such collections were classified under this term); (d) post-necrotic pancreatic/ peripancreatic fluid collections (PNPFC): fluid collections associated with necrotising pancreatitis, containing both fluid and necrotic tissue, which over the course of weeks, evolves into a necrotic fluid collection with both liquid and solid debris; and (e) WOPN: these are formed due to encapsulation of the PNPFC over time in a thickened wall of fibrous or granulation tissue without an epithelial lining at the interface of necrotic and viable tissue; WOPN can be sterile or infected.

The inclusion criteria employed during patient selection for DEN were: (a) suitability for endoscopic transenteric drainage (duration of fluid collection $>4$ weeks, well-formed wall surrounding the collection, accessible endoscopically, located within $1 \mathrm{~cm}$ of the duodenal or gastric walls, and symptomatic); and (b) presence of solid debris within the infected fluid collection. Exclusion criteria for DEN were: (a) APFC or PNPFC; (b) asymptomatic PC; (c) necrosis with minimal liquefaction; (d) walled-off pancreatic collections with no solid debris that were treatable by endoscopic transenteric stenting alone; and (e) no consent for endoscopic interventions.

DEN was performed as a staged procedure using a transgastric approach for all patients due to the proximity of the collection to the gastric wall. EUS-guided transgastric stenting was performed at the first session for initial drainage and to establish endoscopic access to the infected collection. This was similar to standard transgastric stenting for PC drainage. Prior to drainage, all patients were already receiving intravenous carbapenem for treatment. Under conscious sedation using a combination of intravenous midazolam and fentanyl, a linear echoendoscope was used to visualise the infected collection across the gastric wall. Under Doppler ultrasonographic-guidance, the collection was punctured using a 19G needle (EUSN-19-T; Cook Endoscopy, Winston-Salem, NC, USA) (Fig. 1) and a 0.035" guidewire was inserted through the needle into the collection under fluoroscopic

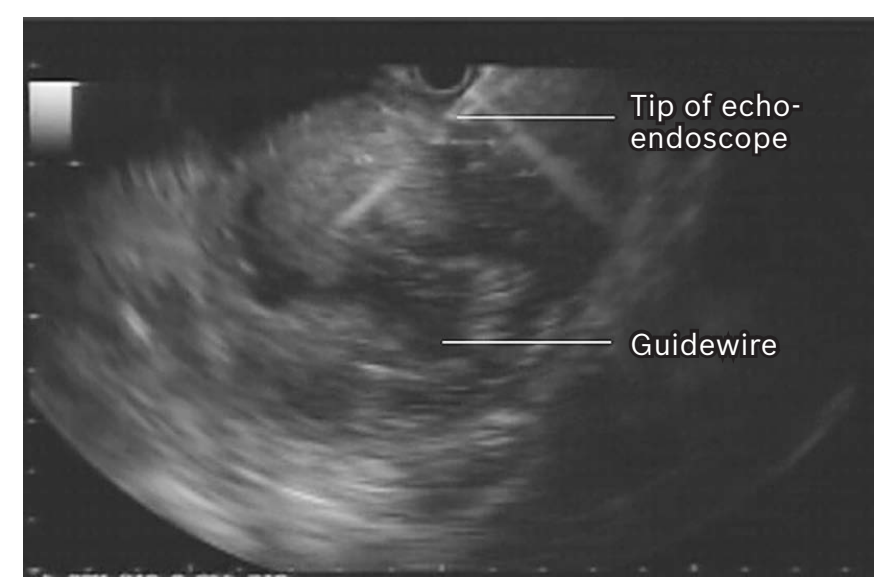

Fig. 1 Endoscopic US image shows an infected pseudocyst being punctured by a $19 \mathrm{G}$ needle.

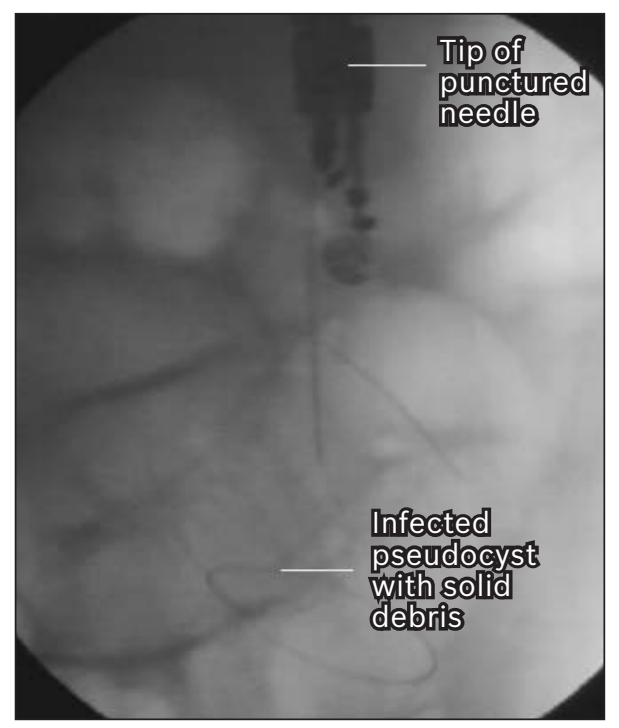

Fig. 2 Radiograph shows a guidewire inserted into the pseudocyst cavity.

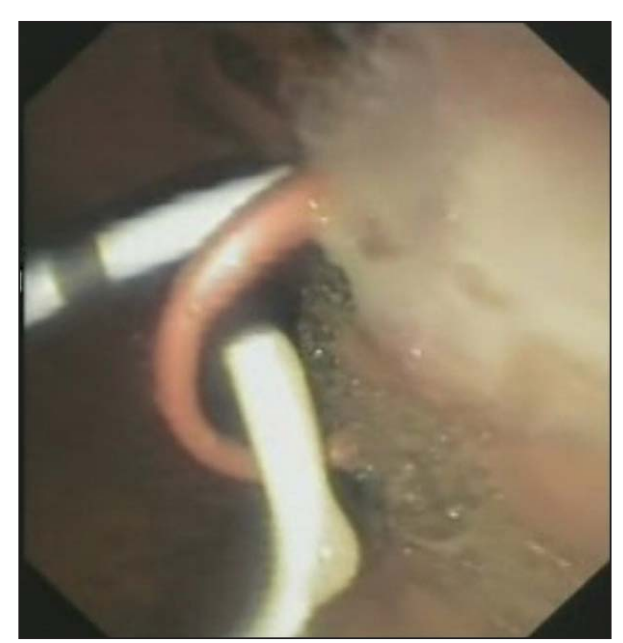

Fig. 3 Endoscopic image shows the transgastric stents inserted for initial drainage.

guidance (Fig. 2). The puncture tract was sequentially dilated to $8-10 \mathrm{~mm}$ using a Soehendra dilator (Cook Endoscopy) and balloon dilators (CRE ${ }^{\mathrm{TM}}$, Boston Scientific, Natick, MA, USA). Two double pigtail stents (both were $10 \mathrm{Fr}$ in diameter, or one was $10 \mathrm{Fr}$ while the other was $8.5 \mathrm{Fr}$ ) were then inserted for transgastric drainage of the infected collection (Fig. 3). The two stents 

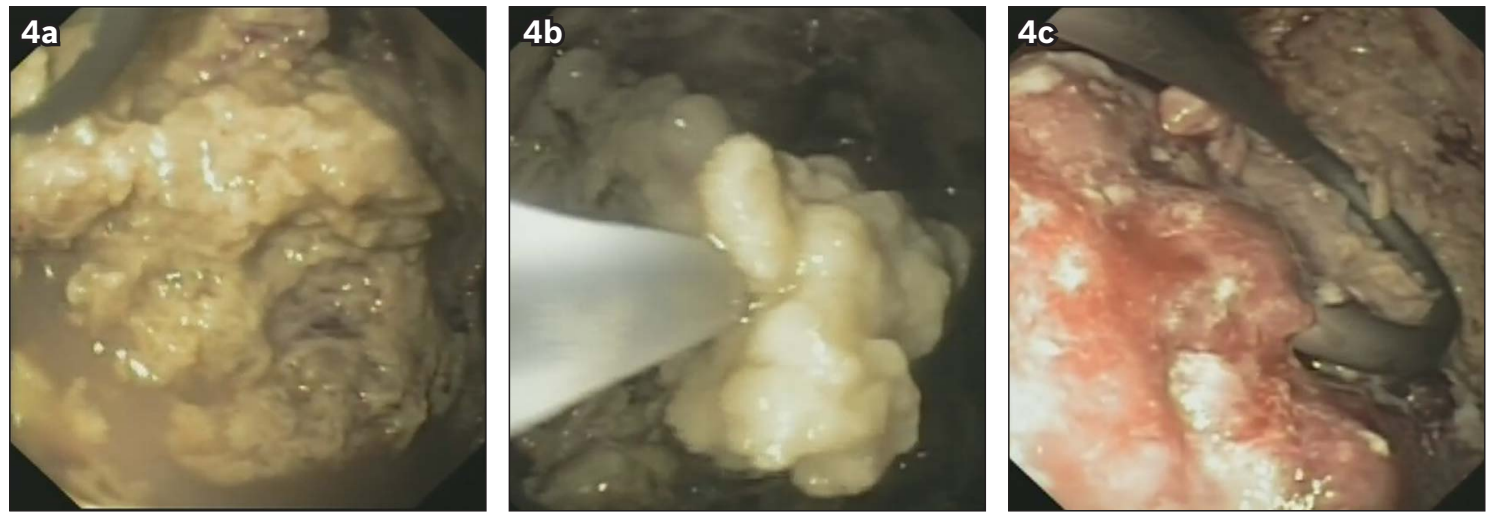

Fig. 4 Endoscopic images show (a) the view inside the infected fluid collection after insertion of the gastroscope; (b) endoscopic necrosectomy being performed using a Dormia basket; and (c) complete removal of all solid debris from the cavity post endoscopic necrosectomy.

\section{Table I. Patient characteristics.}

\begin{tabular}{|c|c|c|c|c|c|}
\hline Patient no. & Age (yrs)/gender & Type of pancreatic collection & Maximum diameter $(\mathrm{cm})$ & Technical success & Clinical success \\
\hline 1 & $41 /$ male & Infected WOPN & 17.2 & Yes & Yes \\
\hline 2 & $23 /$ male & Infected PC with solid debris & 12.0 & Yes & Yes \\
\hline 3 & $54 /$ female & Infected WOPN & 10.3 & Yes & Yes \\
\hline 4 & $34 /$ male & Infected WOPN & 7.8 & Yes & Yes \\
\hline 5 & $69 /$ male & Infected PC with solid debris & 14.2 & Yes & Yes \\
\hline 6 & $64 /$ male & Infected WOPN & 11.1 & Yes & Yes \\
\hline 7 & $63 /$ female & Infected PC with solid debris & 16.2 & Yes & Yes \\
\hline 8 & $47 /$ female & Infected PC with solid debris & 11.3 & Yes & No \\
\hline
\end{tabular}

PC: pseudocyst; WOPN: walled-off pancreatic necrosis

were inserted either sequentially via repeated cannulation of the cavity under fluoroscopy or using a double wire technique. ${ }^{(1,9)}$

The second session was performed after 24-48 hours. Endoscopy was repeated under conscious sedation. The cystogastrostoma was recannulated, a guidewire inserted into the collection and a balloon catheter (CRE ${ }^{\mathrm{TM}}$ ) used to dilate the stoma endoscopically up to $15 \mathrm{~mm}$. A standard gastroscope was then inserted into the cavity (Fig. 4a). Saline irrigation, suctioning and debridement of the necrotic material using Dormia baskets (Fig. 4b) and retrieval nets were performed until all necrotic debris was removed (Fig. 4c). Additional sessions (range 1-3) required for endoscopic necrosectomy were repeated at time intervals of 24-48 hours. Endoscopic necrosectomy was deemed to be complete when the pink granulating wall could be seen with no or minimal debris (Fig. 4c). Two double pigtail transgastric stents were usually left in place to prevent any reaccumulation of fluid collection. These stents were removed endoscopically once follow-up imaging, usually performed within 2-3 months, demonstrated cavity resolution.

Clinical data on patient characteristics, presentation, aetiology, type of endoscopic treatment and outcome were analysed. Outcome data included technical and clinical success rates, as well as complication rates. Technical success was defined as successful completion of DEN. Clinical success was defined as complete clinical resolution with no need for alternative salvage treatment following DEN. Categorical data were summarised as proportions, while continuous data were summarised as means and ranges.

\section{RESULTS}

From November 2006 to October 2011, endoscopic transgastric drainage of symptomatic pancreatic fluid collections was performed for 25 patients. Of these, eight patients who underwent DEN from April 2007 to October 2011 were included in the current study (Table I). The mean age of the patients was 50 (range 23-69) years and the male-to-female ratio was 5:3.

The types of pancreatic fluid collections seen among our patients included infected WOPN $(\mathrm{n}=4)$ and infected PC containing solid debris $(n=4)$. The patients presented with fever and abdominal pain. The presence of walled-off pancreatic collections was confirmed using abdominal computed tomography (CT) prior to endoscopic interventions. The mean maximum diameter of the fluid collection was 12.5 (range 7.8-17.2) $\mathrm{cm}$. The underlying aetiologies included severe necrotising pancreatitis $(n=6)$ and post-pancreatic surgery $(n=2)$.

EUS-guided transgastric stenting, followed by endoscopic necrosectomy, was performed for all eight patients. The median number of sessions of endoscopic necrosectomy was 1 (range 1-3) session in our patients. Technical success was achieved in all eight patients, and no procedure-related complications were encountered. Complete clinical resolution of the infected fluid collection was achieved in 7 (87.5\%) patients. One patient had recurrence of symptomatic infected pancreatic collection within a month of the procedure, possibly due to the spontaneous loss of the transgastric stents. This patient opted for surgical intervention rather than repeat endoscopic treatment. Among the seven patients treated successfully, the mean time to removal of transgastric 
stents was 76 (range 47-102) days. No immediate or delayed procedural complications occurred. Pancreatic duct stenting was required in five patients to treat the associated pancreatic duct disruption. These stents were removed after the resolution of pancreatic duct disruption.

\section{DISCUSSION}

Infected pancreatic fluid collections, such as infected PC (previously termed pancreatic abscess) and infected WOPN, require intravenous antibiotic therapy and interventions to drain the collection in order to effectively treat the sepsis, which may otherwise result in considerable morbidity and mortality. In contrast to WOPN (which are collections containing solid necrotic material) and infected PC (which contain solid purulent debris), PCs are fluid collections devoid of any solid debris. Hence, PCs can be drained successfully using endoscopic and percutaneous approaches. Endoscopic drainage, especially under EUS guidance, is usually preferred as a first-line treatment option, when technically feasible, due to the lower morbidity associated with the procedure when compared to surgical and percutaneous drainage, and because its efficacy is similar to surgery. ${ }^{(1-3)}$ Conversely, WOPN is less amenable to endoscopic or percutaneous treatment due to the nonviable solid components involved. Similarly, infected PC containing solid debris may be suboptimally drained by transenteric stenting alone.

Surgery is traditionally considered the standard treatment when interventions are required. In order to minimise morbidity and increase the success rate of treatment, surgery should be delayed to approximately 3-4 weeks after the onset of disease. This delay will allow the encapsulation and demarcation of the infected necrotic collections. Demarcation facilitates necrosectomy and reduces complications related to drainage and debridement procedures. ${ }^{(10,11)}$ Although definitive, surgical drainage and necrosectomy may result in a prolonged recovery period, the need for repeated interventions, as well as the occurence of external fistulae and abdominal wall hernias. ${ }^{(12,13)}$ For instance, a recent multicentre randomised controlled study $y^{(14)}$ randomly assigned 88 patients with necrotising pancreatitis and suspected or confirmed infected necrotic tissue to undergo either primary open necrosectomy or a step-up approach as treatment. The step-up approach consisted of percutaneous drainage, followed by minimally invasive retroperitoneal necrosectomy, if necessary. The primary endpoint was a composite of major complications (e.g. new-onset multiple-organ failure or multiple systemic complications, perforation of a visceral organ or enterocutaneous fistula, or bleeding) or death. It was found that the minimally invasive step-up approach had lower composite endpoints of major complications or death when compared to open necrosectomy ( $40 \%$ vs. $69 \%, p=0.006) .{ }^{(14)}$ This study indirectly supports the concept that minimally invasive therapeutic interventions are a viable treatment option.

Minimally invasive techniques of necrosectomy avoid open laparotomy and involve debridement via retroperitoneal, laparoscopic or endoscopic approaches. They offer the benefit of maintaining the compartmentalisation of the infected area while reducing microbial burden by not contaminating tissue planes and the peritoneal cavity. They may also reduce fistulas, bleeding and wound complications that are associated with open explorations, which commonly require multiple re-explorations. ${ }^{(15)}$ However, it should also be acknowledged that simultaneous to the emergence of such minimally invasive techniques, open necrosectomy has evolved as well and mortality rates associated with the procedure are now low in specialist centres. ${ }^{(10,16)}$ For instance, Rodriguez et al reported an overall operative mortality of $11.4 \%$. In addition, they found the mortality rate in patients operated 28 days after the onset of the disease to be significantly lower (5.1\%) compared to patients who were operated upon earlier (20.3\%). ${ }^{(10)}$ In a recent analysis of the results of pancreatic necrosectomy in North America, Parikh et al reported a low 30 -day mortality rate of $6.8 \%$ and a morbidity rate of $62 \%{ }^{(16)}$

DEN was recently introduced into clinical practice as a minimally invasive endoscopic technique for the treatment of

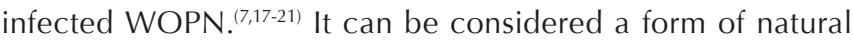
orifice transluminal endoscopic surgery. In our series, we used this technique for the treatment of both infected WOPN and infected PC with solid debris. Comparative studies have demonstrated that for infected pancreatic fluid collections with significant solid debris, endoscopic drainage by insertion of transenteric drains alone was inadequate because the solid debris had to be physically removed; the success rate of endoscopic drainage in such patients could be as low as $25 \%$. $^{(4)}$ A retrospective study, which compared DEN with conventional endoscopic transenteric drainage for the treatment of WOPN, found that successful resolution was accomplished in $88 \%$ of patients who underwent DEN vs. $45 \%$ of those who received standard transenteric drainage $(p<0.01)$, without a change in the total number of procedures. Complications associated with DEN were limited to mild periprocedural bleeding, which occurred at a similar rate as conventional transenteric drainage. ${ }^{(5)}$ To the authors' knowledge, only three large non-comparative case series have been published on DEN so far. These include a German multicentre study $(\mathrm{n}=93),{ }^{(19)}$ a US multicentre study $(\mathrm{n}=104)^{(20)}$ and a large singlecentre case series $(n=80)$ from the centre that initially pioneered this technique. ${ }^{(21)}$ According to these reports, high clinical success rates of $80 \%-91 \%$ could be achieved using DEN. In the German study, the complication and 30-day mortality rates were $26 \%$ and $7.5 \%$, respectively. ${ }^{(19)}$ In the US study, complications occurred in $14 \%$ of patients and included 5 (4.8\%) cases of retrogastric perforations/pneumoperitoneum that were managed nonoperatively. ${ }^{(20)}$

There is a lack of comparative studies between DEN and surgical necrosectomy. To the authors' knowledge, to date, only one published randomised study has compared DEN with surgical necrosectomy. In a recent Dutch multicentre randomised controlled study, ${ }^{(22)}$ patients with signs of infected necrotising 
pancreatitis were randomly assigned to undergo endoscopic ( $n=10)$ or surgical necrosectomy $(n=10)$. Surgical necrosectomy consisted of video-assisted retroperitoneal debridement $(n=6)$ or laparotomy $(n=4)$. The primary endpoint was the post-procedural proinflammatory response, as measured by serum interleukin-6 (IL-6) levels. Secondary clinical endpoints included a composite of death or major morbidity (e.g. newonset multiple organ failure, intra-abdominal bleeding or enterocutaneous fistula) and other morbidities (e.g. pancreatic fistula, new-onset diabetes mellitus or use of pancreatic enzymes). It was found that DEN significantly reduced the post-procedural IL-6 levels compared with surgical necrosectomy. The composite clinical endpoint occurred less often in patients who underwent DEN (20\% vs. $80 \%$; $p=0.03)$. DEN did not cause new-onset multiple organ failure unlike surgery $(0 \%$ vs. $50 \% ; p=0.03)$ and resulted in a lower number of pancreatic fistulas $(10 \%$ vs. $70 \%$; $\mathrm{p}=0.02)^{(22)}$

Although comparative data between DEN and surgery are lacking, the results of surgical series provide some insight into the relative merits of DEN. The outcomes of surgical necrosectomy have recently been reviewed. ${ }^{(15,23)}$ Retroperitoneal necrosectomy was associated with an average major complication rate of $41 \%$ (range $0 \%-43 \%$ ) and a mortality rate of $16 \%$ (range $0 \%-27.3 \%$ ). Meanwhile, laparoscopic necrosectomy was associated with major complication rates of $0 \%-50 \%$ and a mean mortality rate of $7 \%$ (range $0 \%-10.5 \%$ ). However, it must be qualified that the sample size of these individual surgical series was small (retroperitoneal necrosectomy: 1-47 patients; laparoscopic necrosectomy: 1-19 patients), and this may have resulted in outcome bias in these studies. The success and complication rates of the DEN series ${ }^{(19-21)}$ compared favourably with the results reported by the surgical series. ${ }^{(15,23)}$

DEN is technically not feasible if there is minimal liquefaction of the pancreatic necrosis, with predominant solid debris. In such patients, when interventions are required, the main treatment option remains surgical. The presence of splenic vein thrombosis demonstrated by $\mathrm{CT}$ prior to intervention mandates that extra precaution be taken due to the possible occurrence of collateral vessels in the field of endoscopic transgastric access. In this context, Doppler ultrasonographic-guidance during the initial puncture is particularly important. In the context of persistent pancreatic duct disruption, an attempt should be made to stent the pancreatic duct by endoscopic retrograde cholangiopancreaticography (ERCP), in addition to DEN, in order to prevent the recurrence of the fluid collection.

Another relative contraindication is the extension of the necrotic collection beyond the midline across the cava and aorta in the retroperitoneum. DEN is a challenging procedure that should only be performed by experienced therapeutic endoscopists with surgical backup, as severe complications such as perforation, bleeding and embolism may occur. These risks, however, may be minimised by paying meticulous attention to technical details. Cross-sectional imaging such as CT should be used to confirm that the collection is encapsulated by a mature wall and is in close proximity to the gastric lumen before DEN is attempted. Any coagulopathy should be corrected. In our study, care was taken to not dilate the drainage tract larger than $1 \mathrm{~cm}$ at the index endoscopy, and dilating the diameter of the cystogastrostoma to $15 \mathrm{~mm}$ was preferably attempted at the second session in order to reduce the risk of perforation. During the process of endoscopic necrosectomy, over-insufflation of the cavity with air must be avoided, and gentle debridement using saline lavage and aspiration, baskets, soft snares and retrieval nets should be performed. The use of carbon dioxide (if available), rather than air, for insufflation is advisable in order to minimise the theoretical risk of air embolism. One should restrict debridement to the necrotic debris that has detached from the wall rather than forcibly attempting to remove necrotic matter adherent to the wall. Treatment-related perforations might potentially be treated conservatively, as in the US multicentre case series where $5(4.8 \%)$ retrogastric perforations/pneumoperitoneum were managed non-surgically. ${ }^{(20)}$ However, it should be noted that should severe bleeding due to vascular laceration occur, salvage surgery would be required for haemostasis. In our case series, no complications were encountered.

Percutaneous drainage using large-bore catheters has been used by interventional radiologists to manage symptomatic PC. However, such techniques are associated with variable success, a need for prolonged external drainage and the occurrence of local complications such as pancreaticocutaneous fistulae. In addition, it is difficult for such drainage catheters to adequately address the problem of solid debris within infected collections, although this may be circumvented to a certain extent by the use of continuous saline irrigation via these large-bore catheters. Nonetheless, external drainage may still have a role to play as adjunctive treatment for collections that cannot be accessed endoscopically, or for patients who are not stable enough to undergo endoscopy or surgery.

The technique of combined modality treatment (CMT), in which endoscopic transenteric stents are added to a regimen of percutaneous drains, has been introduced to overcome the problem of chronic pancreaticocutaneous fistulae as a result of percutaneous drainage. It is based on the clinical observation that patients with WOPN who spontaneously fistulise into the duodenum during percutaneous drainage have shorter and less complicated hospital courses and no pancreaticocutaneous fistulae. In CMT, a controlled fistula between the necrotic fluid collection and lumen of the bowel is created by transenteric stenting at the beginning of percutaneous drainage. External drains are still necessary to lavage WOPN. A retrospective comparative study found that compared to percutaneous drainage alone, CMT resulted in a significant decrease in length of hospitalisation, duration of external drainage and number of radiological imaging studies. ${ }^{(24)}$ Compared to DEN, CMT avoids the need for large-diameter balloon dilation of the necroenterostomy and endoscopic passage into the retroperitoneum, and may 
theoretically reduce the risk of haemorrhage, perforation and air embolism.

Apart from considering the need and choice of drainage techniques to address the possibility of recurrent pancreatic fluid collections, the pancreatic duct anatomy must also be assessed as part of a holistic treatment strategy. Fluid collections would recur in the presence of persistent pancreatic duct disruption or disconnected pancreatic duct syndrome. ${ }^{(25)}$ Endoscopic treatment by stenting across the duct disruption may facilitate healing and prevent recurrence. ${ }^{(26)}$ When fistulas persist despite prolonged stenting, it is possible to seal the fistula endoscopically using cyanoacrylate. ${ }^{(27,28)}$ When there is complete disruption of the pancreatic duct, as seen in patients with disconnected pancreatic duct syndrome, it may be technically difficult to bridge the disruption endoscopically; such a situation will predispose the patient to recurrent fluid collections. Even when successful, the effect of endoscopic therapy may only be temporary and surgery might still be required. A randomised trial suggested that long-term transenteric stenting in patients with disconnected pancreatic duct syndrome may be an alternative to surgery to prevent the recurrence of collection. ${ }^{(29)}$ However, more data would be required to confirm this finding, given the concerns regarding stent occlusion and infection.

This study was not without its limitations. This was a small, retrospective, single-centre case series that did not compare the outcomes against that of an alternative treatment modality. Nonetheless, the results of our study are noteworthy, as the technique of DEN is currently only available in limited referral centres globally, with limited published data from Europe and the USA. In our study, the technical and clinical success rates were $100 \%$ and $87.5 \%$, respectively. No major complications were encountered in our patients, which was consistent with current published data.

Current data suggest that DEN has the potential to be a less invasive and less risky alternative to open surgical necrosectomy in the management of infected WOPN and infected PC with solid debris. For the management of such complex disease entities, a multidisciplinary team approach is crucial, and surgical and radiological drainage remain important as salvage therapies, or when DEN is technically unfeasible. The management paradigm for such collections has shifted from open laparotomy to minimally invasive treatment options. The final selection of minimally invasive surgical necrosectomy, DEN or CMT will depend on multiple factors, including the expertise available at a given centre, specific patient characteristics and risk assessment findings.

\section{REFERENCES}

1. Seewald S, Ang TL, Teng KC, Soehendra N. EUS-guided drainage of pancreatic pseudocysts, abscesses and infected necrosis. Dig Endosc 2009; 21(1 suppl):S61-5.

2. Seewald S, Ang TL, Kida M, Teng KY, Soehendra N. EUS 2008 Working Group document: evaluation of EUS-guided drainage of pancreatic-fluid collections (with video). Gastrointest Endosc 2009; 69(2 suppl):S13-21.

3. Varadarajulu S, Lopes TL, Wilcox CM, et al. EUS versus surgical cystgastrostomy for management of pancreatic pseudocysts. Gastrointest Endosc 2008; 68:649-55.

4. Hookey LC, Debroux S, Delhaye M, et al. Endoscopic drainage of pancreatic-fluid collections in 116 patients: a comparison of etiologies, drainage techniques, and outcomes. Gastrointest Endosc 2006; 63:635-43.

5. Gardner TB, Chahal P, Papachristou GI, et al. A comparison of direct endoscopic necrosectomy with transmural endoscopic drainage for the treatment of walled-off pancreatic necrosis. Gastrointest Endosc 2009; 69:1085-94.

6. Seifert H, Wehrmann T, Schmitt T, Zeuzem S, Caspary WF. Retroperitoneal endoscopic debridement for infected peripancreatic necrosis. Lancet 2000; 356:653-5.

7. Seewald S, Groth S, Omar S, et al. Aggressive endoscopic therapy for pancreatic necrosis and pancreatic abscess: a new safe and effective treatment algorithm. Gastrointest Endosc 2005; 62:92-100.

8. Bollen TL, Besselink MG, van Santvoort HC, Gooszen HG, van Leeuwen MS. Toward an update of the atlanta classification on acute pancreatitis: review of new and abandoned terms. Pancreas 2007; 35:107-13.

9. Ang TL, Teo EK, Fock KM. EUS-guided drainage of infected pancreatic pseudocyst: use of a 10F Soehendra dilator to facilitate a double-wire technique for initial transgastric access (with videos). Gastrointest Endosc 2008; 68:192-4.

10. Rodriguez JR, Razo AO, Targarona J, et al. Debridement and closed packing for sterile or infected necrotizing pancreatitis: insights into indications and outcomes in 167 patients. Ann Surg 2008; 247:294-9.

11. Besselink MG, Verwer TJ, Schoenmaeckers EJ, et al. Timing of surgical intervention in necrotizing pancreatitis. Arch Surg 2007; 142:1194-1201.

12. Vege SS, Baron TH. Management of pancreatic necrosis in severe acute pancreatitis. Clin Gastroenterol Hepatol 2005; 3:192-6.

13. Uhl W, Warshaw A, Imrie C, et al. IAP Guidelines for the Surgical Management of Acute Pancreatitis. Pancreatology 2002; 2:565-73.

14. van Santvoort HC, Besselink MG, Bakker OJ, et al. A step-up approach or open necrosectomy for necrotizing pancreatitis. N Engl J Med 2010; 362:1491-502.

15. Babu BI, Siriwardena AK. Current status of minimally invasive necrosectomy for post-inflammatory pancreatic necrosis. HPB (Oxford) 2009; 11:96-102.

16. Parikh PY, Pitt HA, Kilbane M, et al. Pancreatic necrosectomy: North American mortality is much lower than expected. J Am Coll Surg 2009; 209:712-9.

17. Papachristou GI, Takahashi N, Chahal P, Sarr MG, Baron TH. Peroral endoscopic drainage/debridement of walled-off pancreatic necrosis. Ann Surg 2007; 245:943-51.

18. Charnley RM, Lochan R, Gray H, et al. Endoscopic necrosectomy as primary therapy in the management of infected pancreatic necrosis. Endoscopy 2006; 38:925-8.

19. Seifert H, Biermer M, Schmitt W, et al. Transluminal endoscopic necrosectomy after acute pancreatitis: a multicentre study with long-term follow-up (the GEPARD Study). Gut 2009; 58:1260-6.

20. Gardner TB, Coelho-Prabhu N, Gordon SR, et al. Direct endoscopic necrosectomy for the treatment of walled-off pancreatic necrosis: results from a multicenter U.S. series. Gastrointest Endosc 2011; 73:718-26.

21. Seewald S, Ang TL, Richter $H$, et al. Long-term results after endoscopic drainage and necrosectomy of symptomatic pancreatic fluid collections. Dig Endosc 2012; 24:36-41.

22. Bakker OJ, Van Sanvoort HC, van Brunschot S, et al. Endoscopic transgastric vs surgical necrosectomy for infected necrotizing pancreatitis: a randomized trial. JAMA 2012; 307:1053-61.

23. Fink D, Soares R, Matthews JB, Alverdy JC. History, goals, and technique of laparoscopic pancreatic necrosectomy. J Gastrointest Surg 2011; 15:1092-7.

24. Gluck M, Ross A, Irani S, et al. Endoscopic and percutaneous drainage of symptomatic walled-off pancreatic necrosis reduces hospital stay and radiographic resources. Clin Gastroenterol Hepatol 2010; 8:1083-8.

25. Nealon WH, Bhutani M, Riall TS, et al. A unifying concept: pancreatic ductal anatomy both predicts and determines the major complications resulting from pancreatitis. J Am Coll Surg 2009; 208:790-9.

26. Telford JJ, Farrell JJ, Saltzman JR, et al. Pancreatic stent placement for duct disruption. Gastrointest Endosc 2002; 56:18-24.

27. Seewald S, Brand B, Groth S, et al. Endoscopic sealing of pancreatic fistula by using N-butyl-2-cyanoacrylate. Gastrointest Endosc 2004; 59:463-70.

28. Ang TL, Teo EK, Fock KM. Endoscopic drainage and endoscopic necrosectomy in the management of symptomatic pancreatic collections. J Dig Dis 2009; 10:213-24.

29. Arvanitakis M, Delhaye M, Bali MA, et al. Pancreatic-fluid collections: a randomized controlled trial regarding stent removal after endoscopic transmural drainage. Gastrointest Endosc 2007; 65:609-19. 